DOI: $10.35643 /$ Info.26.1.14

Artículo original

\title{
Aprendizaje-Servicio en los estudios de Información y Documentación: una experiencia con personas mayores
}

\author{
Service Learning in Library and Information Science: an experience with \\ elderly people \\ Serviço de aprendizagem em estudos de informação e documentação: uma \\ experiência com pessoas mais velhas
}

\author{
Michela Montesi $^{\mathrm{a}}$ \\ Isabel Villaseñor Rodríguez \\ María Antonia García Moreno ${ }^{\mathrm{c}}$
}

\begin{abstract}
a Departamento de Biblioteconomía y Documentación, Facultad de Ciencias de la Documentación, Universidad Complutense de Madrid ORCID: 0000-0002-5509-2075. Correo electrónico: mmontesi@ucm.es.
\end{abstract}

b Departamento de Biblioteconomía y Documentación, Facultad de Ciencias de la Documentación, Universidad Complutense de Madrid. ORCID: 0000-0002-6000-7824. Correo electrónico: $\underline{\text { isavilla@ucm.es. }}$

c Departamento de Biblioteconomía y Documentación, Facultad de Ciencias de la Documentación, Universidad Complutense de Madrid. ORCID: 0000-0002-2369-5488. Correo electrónico: mariaant@ucm.es.

\section{Resumen}

Se presentan los resultados de un proyecto de Aprendizaje y Servicio (ApS) en el Grado en Información y Documentación (I\&D) de la UCM realizado en colaboración con dos Centros de Mayores del Ayuntamiento de Madrid en el periodo de febrero a junio de 2018 y de 2019. Se describe la metodología docente del ApS, las razones formativas para implantarla en el grado de I\&D y las implicaciones institucionales y sociales de este tipo de iniciativas. Entre los resultados, se analizan los datos de dos encuestas suministradas al alumnado participante tras la finalización del proyecto en el curso 2017-18 y 2018-19, y se resumen además los temas más destacados de un grupo focal con las personas mayores participantes. Desde el punto de vista de la formación, se destaca el avance importante en competencias transversales relacionadas con la comunicación, así como la adquisición de conocimientos y habilidades difíciles de trabajar en las aulas, como la consciencia de los mecanismos de inclusión y exclusión informacional de la población mayor. En cuanto al impacto en la 
comunidad que ha recibido el servicio, la población mayor de los dos centros, se destacan positivamente el marco participativo de las actividades, la calidad humana del alumnado UCM y la esencialidad para la vida diaria de los contenidos impartidos en los talleres.

Palabras clave: APRENDIZAJE Y SERVICIO; COMPETENCIAS INFORMACIONALES; COMPETENCIAS DIGITALES; VALORES CIUDADANOS; NECESIDADES DE INFORMACIÓN; FORMACIÓN DE USUARIOS; ENVEJECIMIENTO POBLACIONAL; PERSONAS MAYORES; MADRID.

\begin{abstract}
The article presents the results of a Service Learning (SL) project within the undergraduate program in Information and Documentation (I\&D) at the Complutense University of Madrid. The project was carried out in collaboration with two Senior Centres of the city of Madrid in the period of February to June during the academic years 2017-18 and 2018-19. We describe SL as an experiential teaching method, the curricular reasons for implementing it, and the institutional and social implications of this initiative. Among the results, we analyse data from two surveys which were administered to students at the end of the project in 2017-18 and 2018-19, and we summarize the main themes of a focal group with participating elderlies. From the curricular point of view, improvements were observed in general competencies related to communication, as well as in the acquisition of knowledge and abilities which are difficult to develop in the classroom, such as becoming aware of the dynamics of information exclusion of elderly people. As for the impact on the community that received the service, the elderly participants judged positively the participatory character of the activities, the human qualities of UCM students, and the essentiality of the contents taught within the courses.
\end{abstract}

Keywords: SERVICE LEARNING; INFORMATION COMPETENCIES; DIGITAL COMPETENCIES; CIVIC VALUES; INFORMATION NEEDS; USER TRAINING; AGING; ELDERLIES; MADRID.

\title{
Resumo
}

Os resultados de um projecto de Aprendizagem e Serviço (ApS) do Curso de Licenciatura em Informação e Documentação (I\&D) da UCM, realizado em colaboração com dois Centros de Terceira Idade da Câmara Municipal de Madrid, são apresentados no período de Fevereiro a Junho 2018 e 2019. São descritas a metodologia de ensino da ApS, os motivos da formação para a sua implementação no curso de I\&D e as implicações institucionais e sociais deste tipo de iniciativas. Dentre os resultados, são analisados os dados de duas pesquisas fornecidas aos alunos participantes após o término do projeto nos anos letivos 2017-18 e 201819 , sendo também analisados os temas de maior destaque de um grupo focal com os idosos participantes resumido. Do ponto de vista da formação, destaca-se o importante avanço nas competências transversais relacionadas com a comunicação, bem como a aquisição de conhecimentos e habilidades difíceis de trabalhar em sala de aula, como a consciência dos mecanismos de inclusão e exclusão de informações da população idosa. Relativamente ao impacto na 
comunidade que recebe o serviço, a população idosa dos dois centros, o enquadramento participativo das actividades, a qualidade humana dos alunos da UCM e a essencialidade para o quotidiano dos conteúdos ministrados nas oficinas. Palavras-chave: APRENDIZAGEM DE SERVIÇOS; COMPETÊNCIAS DE INFORMAÇÃO; COMPETÊNCIAS DIGITAIS; VALORES CÍVICOS; NECESSIDADES DE INFORMAÇÃO; TREINAMENTO DE USUÁRIO; ENVELHECIMENTO; IDOSOS; MADRID.

Fecha de recibido: $15 / 12 / 2020$

Fecha de aceptado: 17/03/2021

\section{Introducción}

En el presente trabajo se exponen los resultados de una experiencia docente de Aprendizaje y Servicio (ApS) en el ámbito del itinerario formativo del Grado en Información y Documentación (I\&D) de la Universidad Complutense de Madrid (UCM), haciendo hincapié en las implicaciones formativas de la experiencia, así como en el impacto de la misma en el entorno social en el cual ha tenido lugar. Concretamente, el proyecto se ha realizado durante los cursos académicos 2017 18 y 2018-19 en colaboración con dos Centros de Mayores del Ayuntamiento de Madrid ubicados en el distrito de Chamberí, implicando a un colectivo de aproximadamente 120 estudiantes y 360 personas mayores. El objetivo de este artículo es mostrar las ventajas de esta metodología docente para los estudios de I\&D y su potencial de impacto en la comunidad a la que se presta el servicio, en este caso personas mayores, para sentar las bases de futuras iniciativas.

\subsection{El Aprendizaje y Servicio para la formación en Información y Documentación}

El ApS, conocido también como Aprendizaje y Servicio Solidario (Tapia y Montes, 2016) es una forma de educación experiencial basada en la prestación de servicios a la comunidad, cuyo objetivo es promover el aprendizaje y desarrollo del alumnado a través de la resolución de problemas del mundo real (Riddle, 2003). Como herramienta pedagógica, además de su carácter experiencial, se caracteriza por tres aspectos fundamentales: (1) la reciprocidad o la colaboración y aprendizaje mutuo entre estudiantes y comunidades, (2) la educación cívica o el desarrollo del sentido de responsabilidad social y (3) la reflexión sobre la 
experiencia realizada (Kott, 2016). Manzano, citado en (Rodríguez-Gallego, 2014), y Folgueiras, Luna y Puig (2013) añaden como cuarto aspecto el peso presencial de los estudiantes.

La metodología del ApS, por definición, es aplicable en prácticamente todo contexto educativo, aunque desconocemos experiencias parecidas en la formación superior en I\&D en España, donde las competencias informacionales en la educación universitaria se han trabajado más bien desde el ámbito de la Educación, por ejemplo, en (Amat, 2011), (Juarros et al., 2012) y (Rubio i Serrano et al., 2013). Las experiencias de ApS en universidades latinoamericanas que recopila el Centro Latinoamericano de Aprendizaje y Servicio Solidario (CLAYSS) tampoco incluyen iniciativas en el I\&D (Ochoa, 2014). En el contexto estadounidense, por otro lado, sí existen numerosas experiencias de aplicación del ApS a la formación en I\&D, tanto en la enseñanza presencial como en la virtual, y como estrategia docente aplicable desde las etapas más tempranas de la formación especializada y de forma complementaria a las prácticas curriculares (Most, 2011). La monografía publicada en 2009 por la American Libraries Association sobre ApS recopila proyectos que han empleado esta metodología de aprendizaje experiencial para la formación universitaria en I\&D (Roy, Jensen, y Meyers, 2009), mientras que en (Nutefall, 2016) se recogen experiencias de ApS realizadas en colaboración con bibliotecas académicas. Según Riddle (2003), el ApS sería especialmente relevante para los estudios en $I \& D$, porque entre los beneficios derivados de esta metodología estarían una percepción diferente de la información y una mayor conciencia de las necesidades de información que plantea la resolución de problemas reales. Montiel Overall (2010) reitera las ventajas del ApS para la formación en I\&D, indicando que los alumnos participantes en un proyecto con escuelas y bibliotecas públicas, relacionado con la brecha digital y la diversidad, se vieron más motivados a analizar las lecturas de clase. La relevancia del ApS para los estudios de I\&D se ve reforzada, además, por sus diferentes características respecto al practicum, más tradicional en estos estudios. Según Angel (2016), sería la reflexión individual durante la experiencia que diferencia claramente el $\mathrm{ApS}$ de las prácticas curriculares. Las prácticas curriculares en la carrera de Biblioteconomía y Documentación responden a la 
necesidad de aprender a intermediar con las personas (Espelt y Mañà, 2011), sin embargo, suelen darse en ámbitos institucionales o empresariales, limitando la adquisición de valores a estos mismos ámbitos. Por otro lado, el ApS busca desarrollar valores cívicos y aportar beneficios mutuos al alumnado y la comunidad (Lim y Bloomquist, 2015). Además, la experiencia del practicum del Grado en I\&D de la Universidad Carlos III de Madrid apunta a que, entre las varias competencias adquiridas por los alumnos en sus prácticas curriculares, la que registra la nota más baja es la capacidad de análisis y síntesis (Pérez-Lorenzo, Vianello Osti, y Pacios Lozano, 2013), siendo, en cambio, la reflexión central para el ApS. Finalmente, no solo es importante diferenciar el ApS del prácticum sino de otro tipo de iniciativas con clara intencionalidad solidaria como el voluntariado, pues en el ApS se persiguen simultáneamente objetivos de servicio y objetivos de aprendizaje (Rodríguez-Gallego, 2014).

\subsection{El ApS para promover valores cívicos y el compromiso social}

En el contexto de la presente experiencia, el ApS se adoptó como una estrategia para promover los valores cívicos y el compromiso social, por un lado, y compensar ciertas limitaciones en la oferta formativa en I\&D, por otro. Entre las otras limitaciones, cabe mencionar la concepción de la carrera en I\&D principalmente como técnica, enfatizando el conocimiento de técnicas como la catalogación, la clasificación o la indización, o del manejo de herramientas tecnológicas. Al mismo tiempo, la relación con las personas destinatarias de servicios de información se suele contemplar desde el punto de vista de las organizaciones dentro de las cuales se ofrecen, a menudo como una cuestión de satisfacción con los servicios bibliotecarios o documentales, mientras que las necesidades de información y el uso de la información se dan tanto dentro de centros de documentación y bibliotecas como en cualquier otro entorno de la vida diaria (Villaseñor-Rodríguez, 2017). En el Libro Blanco del Título de Grado en Información y Documentación (2004), la lista de 23 competencias específicas del grado en I\&D se abre con la "Interacción con los productores, los usuarios y los clientes de la información", contextualizando la relación con las personas consumidoras de información en el ámbito de instituciones y empresas, mientras que trece de las restantes competencias se refieren de forma explícita a técnicas y 
tecnologías. Por otro lado, varias de las competencias nucleares que recopila, para la formación bibliotecaria, la American Library Association (2009) enfatizan las dimensiones comunicativas e interpersonales, así como la implicación social de la profesión, desde métodos para interactuar con personas de todas las edades y grupos, hasta la capacidad de responder a diferentes necesidades y comunidades usuarias. Jardine (2016) recuerda que, sin las personas como referentes, bibliotecas, archivos y centros de documentación no serían sino colecciones de datos en estructuras físicas y añade que en la formación bibliotecaria es necesario incluir también unas "competencias culturales", que permitan trabajar y suministrar servicios a comunidades cada vez más diversas, en términos de raza, edad, género, idioma, nivel económico y mucho más. Aunque las necesidades de formación técnica siguen representando una prioridad formativa para las exigencias del mercado laboral actual (Tejada-Artigas et al., 2014), el suministro de servicios informacionales adecuados exige desarrollar competencias sociales y comunicativas para conectar con el público de referencia incluso fuera de bibliotecas y centros de documentación específicos, más teniendo en cuenta que existen carencias de formación para gestionar en la práctica profesional la “atención al cliente" (Colón-Aguirre, 2017). Finalmente, las competencias interpersonales parecen imprescindibles para realizar tareas más complejas y colaborativas tales como la gestión de datos, por lo menos según la encuesta realizada por (Mizzy y Hayslett, 2016) con profesionales activos en este sector.

Otra consecuencia del énfasis en la formación técnica y en las necesidades organizativas por encima de las necesidades de las personas es que se infravaloran los valores cívicos, tan necesarios para afrontar los desafíos de la sociedad actual. La metodología del ApS busca precisamente, con palabras de Roser Batle (s.f.), poner en práctica los conocimientos adquiridos en los estudios para responder a necesidades reales del entorno. El objetivo final es que el alumnado aprenda a reconocer estas necesidades, a través de una mayor empatía o sensibilidad hacia un grupo poblacional concreto, tendiendo puentes hacia colectivos a menudos afligidos por la soledad, como las personas mayores (Sánchez-Esparza, 2018). Además, los beneficios del ApS como metodología docente están comprobados precisamente en las dimensiones afectivas, incluyendo una mayor autoestima, 
mayor responsabilidad social y mayor autoeficacia (Rodríguez-Gallego y Ordóñez-Sierra, 2015), mientras que desde un punto de vista cognitivo los resultados obtenidos apuntan solo en ocasiones a una ventaja del ApS frente a otras estrategias en la adquisición de conocimiento (Riddle, 2003).

\subsection{El significado del ApS para las instituciones de enseñanza superior}

Muchos de los beneficios del ApS derivan de su vinculación con la comunidad (Rodríguez-Gallego, 2014) y la interacción entre alumnado y diferentes comunidades implica un alto potencial de transformación social (Aramburuzabala, 2013). De esta manera el interés por promover los valores cívicos y el compromiso social típico del ApS converge con un objetivo de mayor trascendencia que atañe a las propias instituciones de educación superior (Montesi y Villaseñor Rodríguez, 2018). La "tercera misión" de la universidad se ha entendido tradicionalmente como la capacidad de transferencia a la sociedad de conocimiento científico para el avance económico y tecnológico (Miettinen, Tuunainen, y Esko, 2015). Sin embargo, cada vez más emerge una nueva visión de esta tercera misión orientada hacia los retos de sostenibilidad que plantea el presente y que exige que la universidad asuma un nuevo rol, el de transformadora social y co-creadora de conocimiento, colaborando con actores diferentes a los tradicionales interlocutores académicos y empresariales (Trencher, Yarime, McCormick, et al., 2013). El análisis de 39 colaboraciones universidad - sociedad en varios países del mundo más desarrollado, permite concluir a Trencher, Yarime, McCormick, et al., 2013 que el ApS es una de las propiedades clave de la co-creación para la sostenibilidad, al igual que la investigación acción, la transferencia de conocimiento, la transdisciplinaridad o los laboratorios vivientes, entre otras. Montague, Wolske, y Larkee (2009), quienes analizan el ApS desde la perspectiva de la administración universitaria, el profesorado y los estudiantes, denominan la tercera misión directamente "servicio", estableciendo una conexión entre la tercera misión y el servicio que se presta en el marco de un proyecto de ApS. Thompson y Hood (2016), quienes tratan el ApS en el marco de las relaciones entre universidad y comunidad, también insisten en la función del ApS en apoyar a las instituciones de educación superior en el desempeño de su "servicio" o tercera misión. Conciben el ApS 
como el resultado de las colaboraciones entre universidad y comunidad y una oportunidad para dejar un impacto importante en comunidades locales y en la sociedad en general. Kott (2016) enfatiza el carácter transformador de las experiencias de ApS, tanto para alumnado, como para el profesorado y otros participantes (Nutefall, 2016; Montague et al., 2009). Según Marullo y Edwards (2000), toda experiencia de colaboración entre universidad y comunidad para afrontar problemas sociales, políticos, económicos o morales permitiría poner en práctica la ciencia de la participación (scholarship of engagement) frente a la más establecida ciencia del descubrimiento (scholarship of discovery), asignando a la universidad un rol más decisivo en la transformación social y valorizando dimensiones normalmente infravaloradas del quehacer académico, es decir la pedagogía, la integración de conocimientos, la interdisciplinaridad, y la aplicación de los mismos. Tapia (2008) afirma que, en el ámbito de la universidad, se ha evolucionado desde el concepto tradicional de extensión universitaria al de responsabilidad social universitaria y que todos los programas de ApS son, por definición, una manifestación de responsabilidad social.

\subsection{El envejecimiento poblacional como reto social}

La adopción de la metodología del ApS tiene implicaciones para la formación especializada en I\&D y las instituciones universitarias, pero además permite abordar problemáticas de tipo social. Específicamente, el reto social que pretende afrontar el proyecto es el del envejecimiento progresivo de la sociedad, abordando un tema que cuenta con escasos ejemplos de ApS, por lo menos en España, donde las experiencias realizadas se desarrollan fuera de la universidad y en relación con asuntos que no están relacionados con la adquisición de competencias informacionales (Batlle \& Escoda, 2019; Grup de Recerca en Educació Moral \& Centre Promotor de l'Aprenentatge Servei, 2019). La adquisición de competencias informacionales, sin embargo, es un problema de gran trascendencia en el contexto de envejecimiento poblacional que caracteriza las sociedades actuales (Pino Juste, Soto Carballo y Rodríguez López, 2015) y según ha quedado de manifiesto en la pandemia por Covid-19 de 2020 (Naciones Unidas, 2020). Aunque en los últimos años, la investigación sobre el tema ha pasado del interés por las posibilidades que la población mayor tenía de acceder a 
las TIC, o la "primera brecha digital" (Abad Alcalá, 2014), al estudio del uso e intensidad que hace de las mismas, o "segunda brecha digital", sabemos muy poco sobre las razones y motivaciones de los adultos mayores para adoptar las TIC, o sobre sus dificultades (Pino Juste, Soto Carballo y Rodríguez López, 2015). En este sentido, los Informes de la Sociedad Digital de Telefónica (2017; 2018) apuntaban a la necesidad de formación continua de este colectivo, que cada vez utiliza más la tecnología para actividades de la vida cotidiana, alertando de un posible riesgo de exclusión digital. La mayoría de las personas consultadas para el Informe de 2018 señalaba como principal motivación para hacer un mayor uso de las aplicaciones que "lo haría si alguien le enseñara" (2018:111), apuntando a la importancia de conectar con el resto del tejido social. Finalmente, la reducción de la brecha digital en los últimos años se debe más a la llegada a estas edades de personas que ya utilizaban internet previamente, que por un trabajo de formación y alfabetización digital de los ya mayores (Abellán-García, 2019:36), y de integración de la población mayor en la sociedad. El uso de internet y las tecnologías contribuye al envejecimiento activo, promoviendo la participación social, la salud, una actitud de cambio y estímulo intelectual (Miguel y Luz, 2017), y aportando beneficios informativos, comunicativos, transaccionales/administrativos y de ocio/entretenimiento (Llorente Barroso, Viñarás Abad y Sánchez Valle, 2015; Abad Alcalá et al, 2017). Las tecnologías, además, ayudarían a sobrellevar la soledad según emerge de los datos del Observatorio Nacional de las Telecomunicaciones y la SI, según el cual la población con mayor crecimiento en el acceso a internet en 2018 fue entre las personas viudas y entre aquellas con 65 a 74 años, con más de 6 puntos porcentuales de incremento (ONTSI, 2018).

Sin embargo, igual de importante que la formación en competencias digitales de la población mayor es la creación de conexiones entre las personas mayores y el resto de la sociedad, con el objetivo de derrumbar prejuicios y discriminaciones basadas en el "edadismo" o "el conjunto de actitudes, creencias, estereotipos, prácticas individuales e institucionales discriminatorias y negativas hacia las personas de más edad" (Ayuntamiento de Madrid. Dirección General de Personas Mayores y Servicios Sociales, 2019: p. 5), y potenciar la conectividad social de un 
colectivo acostumbrado a contar con fuentes de información "personales" y a dirigirse a familiares y amistades para satisfacer sus necesidades informativas (González-Teruel y Andreu-Ramos, 2013). En este sentido, aun enfatizando las competencias digitales, el proyecto objeto de esta contribución busca antes de todo conectar dos extremos generacionales trabajando la integración de la población mayor en la sociedad a través de encuentros e intercambios informacionales entre jóvenes y mayores.

\subsection{El proyecto}

Con base en estos fundamentos teóricos, el proyecto definió como objetivos básicos, por un lado, el desarrollo de competencias informacionales, tales como el manejo de las TIC, la búsqueda de información para el día a día, o la evaluación de la información, en la población mayor del distrito de Chamberí, donde se encuentra ubicada la Facultad de Ciencias de la Documentación y, por otro, competencias sociales y comunicativas, y valores en el alumnado del Grado en I\&D de la propia Facultad. En la ejecución del proyecto participó el alumnado de segundo, tercero y cuarto grado de los cursos académicos 2017-18 y 2018-19, con un total de 120 alumnas y alumnos, abarcando transversalmente 6 asignaturas. La ejecución efectiva de las actividades, que consistieron básicamente en la planificación y ejecución de una serie de talleres, tuvo lugar en el periodo febreromayo de los dos cursos, afectando aproximadamente a 360 personas mayores. Solo se llegaron a impartir un total de 19 talleres de los 31 que se planificaron a lo largo de todo el curso académico (septiembre a mayo), pues algunos de estos se propusieron al centro terminada ya la temporada de actividades para personas mayores.

Cuadro 1. Datos generales relativos al proyecto

\begin{tabular}{|l|c|c|c|}
\hline & $\mathbf{2 0 1 8}$ & $\mathbf{2 0 1 9}$ & Total \\
\hline $\mathrm{N}^{\mathrm{o}}$ talleres & 11 & 20 & 31 \\
\hline $\mathrm{N}^{\mathrm{o}}$ Alumnas/os & 52 & 68 & 120 \\
\hline $\mathrm{N}^{\mathrm{o}}$ Personas mayores & 212 & 146 & 358 \\
\hline $\mathrm{N}^{\mathrm{o}}$ de asignaturas & 4 & 6 & \\
\hline
\end{tabular}




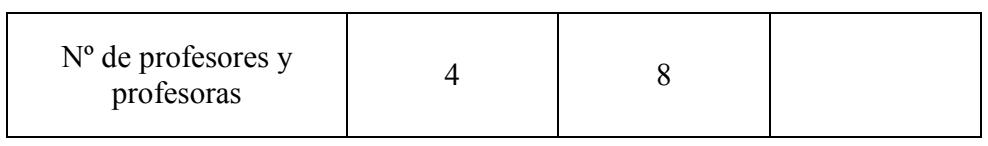

Fuente: Elaboración propia

Los talleres planificados y/o impartidos giraron principalmente alrededor de temáticas culturales y de entretenimiento, por un lado, y tecnológicas, por otro (Cuadro 2). En cultura y entretenimiento, los talleres trataron temáticas tales como la jubilación en China, la historia divertida a través de películas y material de internet, o se organizaron entrevistas, tertulias o juegos de memoria. Entre las actividades relacionadas con la tecnología, se pueden mencionar, como ejemplos, la creación de un blog para el taller de marquetería, los cursos para aprender a utilizar smartphones y aplicaciones móviles, para descargar música de internet y para realizar compras por internet.

Cuadro 2. Temáticas de los talleres impartidos

\begin{tabular}{|l|c|c|c|}
\hline Temática de los talleres & 2018 & 2019 & total \\
\hline Cultura y entretenimiento & 5 & 8 & 13 \\
\hline Tecnologías & 4 & 6 & 10 \\
\hline Vida diaria y salud & 1 & 3 & 4 \\
\hline Otra & 1 & 3 & 4 \\
\hline total & 11 & 20 & 31 \\
\hline
\end{tabular}

Fuente: Elaboración propia

Para poder evaluar la actividad, el alumnado participante plasmó en forma de diario su experiencia y las respectivas reflexiones.

La organización y gestión del proyecto corrió a cargo de un equipo de 8 profesoras y profesores y de la coordinación de los Centros de Mayores (dirección y animadoras sociales), sin tener en cuenta que, en la Comunidad de Madrid, los proyectos de ApS en contextos universitarios están amparados por un acuerdo entre el Ayuntamiento de Madrid y las universidades públicas del espacio madrileño. Tanto por parte de las unidades que coordinan el ApS en las diferentes universidades, como por parte del Ayuntamiento se reciben direcciones y se organizan encuentros, que, aunque menos directamente, repercuten en el 
desarrollo de los proyectos. Esto sirva para transmitir la extrema complejidad del entramado humano que mueve un proyecto de ApS como el presente.

\section{Metodología}

Los datos que se recogieron en el proyecto son numerosos, aunque aquí nos centramos en los siguientes: a) una encuesta con el alumnado realizada al finalizar el proyecto en los dos cursos académicos (mayo de 2018 y mayo de 2019); y b) un grupo focal con las personas mayores participantes con el objetivo de medir el impacto social de las actividades realizadas.

Cabe añadir aquí que la investigación sobre el impacto de los proyectos de ApS es escasa sobre todo por lo que concierne el impacto en las comunidades o impacto social (Chupp y Joseph, 2010). Cronley, Madden, y Davis (2015) subrayan que tradicionalmente el ApS se ha medido desde la perspectiva del alumnado y en todo caso de las propias instituciones, mientras que la poca investigación sobre las comunidades ha asumido una actitud "colonial" hacia las mismas, que se han concebido en términos de "objetos", enfatizando su disponibilidad para la implicación en los proyectos. El énfasis en el impacto académico de los proyectos de ApS ha permitido justificar la viabilidad pedagógica de esta metodología docente en la enseñanza universitaria (James y Logan, 2016). Sieh y Frank (2018), quienes se centran en la metodología del student-community engagement, apuntan a la práctica ausencia de investigación acerca de los efectos de las estrategias basadas en aprendizaje experiencial en la comunidad, incluso cuando se conciben desde un punto de vista institucional. El presente proyecto adolece de estas limitaciones, pues ha enfatizado la medición del impacto pedagógico del mismo frente a la medición del impacto en la comunidad que cuenta exclusivamente con los resultados de un grupo focal.

\subsection{Encuesta}

La encuesta dirigida a las alumnas y alumnos participantes se envió al finalizar el proyecto, tanto en la edición de 2017-18 como en la de 2018-19. Se recogieron un total de 49 respuestas (29 para 2017-18 y 20 para 2018-19), lo que supone una tasa de respuesta de aproximadamente el 40,8\%. Tomaron parte en la encuesta un 
$60 \%$ de alumnas frente a un $40 \%$ de alumnos, cursando en su mayoría el segundo curso del Grado en I\&D (90\%). La participación en el proyecto se realizó principalmente en grupo (85\%) y, en menor medida, de forma individual (17\%).

El cuestionario consistía en 18 preguntas cerradas y abiertas relacionadas con diferentes aspectos de la experiencia, y concretamente: la actitud de los participantes, las competencias trabajadas, la percepción de la experiencia, gustos en cuanto al tipo de formación recibida y expectativas profesionales, además de los datos demográficos que indicamos arriba.

Para algunas de las variables analizadas, concretamente las de experiencia y competencias, realizamos la correspondiente prueba del Chi Cuadrado, con el objetivo de detectar posibles asociaciones entre dimensiones de la experiencia y avance en diferentes competencias. El procesamiento estadístico se realizó a través del software IBM SPSS Statistics 25 y Excel.

\subsection{Grupo focal}

Al finalizar el proyecto, para las dos ediciones, se convocaron reuniones con el objetivo de evaluar el impacto que las actividades habían tenido en la población mayor a través de un grupo focal. Solo para la edición de 2018-19 fue posible realizar el grupo focal, porque en la primera ocasión no se consiguió reunir a un número significativo de participantes y solo se obtuvo una retroalimentación informal. La problemática de involucrar las comunidades objeto del servicio es conocida en la literatura, y James y Logan (2016) mencionan el hecho de que las comunidades pueden ser colectivos amplios y difíciles de monitorizar, existen dificultades de tipo burocrático y puede haber recelos a la hora de participar en procesos de evaluación investigadora. El grupo focal correspondiente a la edición 2019 del proyecto se realizó el día 12 de junio de 2019 en el Centro de Mayores de Santa Engracia. Participaron tres profesoras adscritas al proyecto, una de las animadoras y 12 personas mayores que habían acudido previamente a varios de los talleres impartidos por el alumnado de la Facultad. La duración de la reunión fue de aproximadamente 2 horas y el debate se grabó entero en un archivo de audio. Las personas intervinientes dieron su opinión sobre los talleres impartidos 
y las temáticas de los mismos, y refirieron acerca de su experiencia de participación.

\subsection{Otros datos}

Cabe mencionar que la participación en el proyecto para las autoras de este trabajo ha representado de por si una valiosa fuente de información y datos. Tales experiencias se encuentran analizadas y detalladas, en forma de diarios, en la monografía (Cristóbal Querol, García Moreno, Montesi et al., 2020), a la que remitimos para mayores particulares. Aquí cabe constatar que la ejecución del proyecto supuso la realización de visitas periódicas a los espacios del Centro de Mayores y de reuniones con la dirección, el equipo de animación y miembros de la junta directiva del mismo. Además, dos profesoras del equipo impartieron talleres con personas mayores en el propio centro. Todo esto se ha producido durante un periodo de más de dos años desde finales de 2017 hasta la actualidad (finales de 2019). Este nivel de inmersión en el entorno del centro de mayores permite caracterizar la experiencia como etnográfica $y$, aunque los datos correspondientes a esta experiencia etnográfica no se relaten enteramente aquí, los resultados reflejados en este artículo se ven condicionados por la misma.

\section{Resultados}

\subsection{Encuesta}

\section{Preferencias acerca del tipo de formación}

Para poder contextualizar la experiencia de ApS en el marco de las preferencias formativas de los participantes, se les preguntó, a través de preguntas de escala semántica diferencial, que describieran el tipo de formación que les gusta a través de binomios de adjetivos opuestos o semi-opuestos (Cuadro 1). En una escala del 1 al 7, indicaron si sus preferencias se acercaban más a un extremo u otro de los dos binomios (en el Cuadro 1 está reflejada la mediana de las puntuaciones globales). Los resultados muestran que, aunque en algunos casos vemos una ligera desviación del punto medio (el 4), en general las puntuaciones se sitúan precisamente en el punto medio o cerca de este, hecho que se puede interpretar 
como una exigencia de variedad en las estrategias formativas deseadas por este colectivo estudiantil.

Cuadro 3. Preferencias de los participantes con respecto al tipo de formación. En la tabla, se refleja la mediana de las puntuaciones asignadas en una escala del 1 al 7.

\begin{tabular}{|c|c|c|c|c|c|c|c|c|}
\hline & 1 & 2 & 3 & 4 & 5 & 6 & 7 & \\
\hline Experiencial & & & - & & & & & Teórica \\
\hline $\begin{array}{l}\text { General y aplicable a } \\
\text { diferentes casos }\end{array}$ & & & & & - & & & $\begin{array}{r}\text { Aplicada a casos } \\
\text { concretos } \\
\text { del mundo real }\end{array}$ \\
\hline $\begin{array}{l}\text { Enriquecedora en } \\
\text { competencias técnicas }\end{array}$ & & & & - & & & & $\begin{array}{r}\begin{array}{r}\text { Enriquecedora en } \\
\text { valores }\end{array}\end{array}$ \\
\hline $\begin{array}{l}\text { Activista y } \\
\text { comprometida } \\
\text { socialmente }\end{array}$ & & & & - & & & & $\begin{array}{r}\text { Promotora de } \\
\text { desarrollo } \\
\text { Profesional }\end{array}$ \\
\hline Autónoma & & & & - & & & & Supervisada \\
\hline Grupal & & & & - & & & & Individual \\
\hline Monodisciplinaria & & & & & - & & & $\begin{array}{r}\text { Multidisciplinaria } \\
\text { y transversal }\end{array}$ \\
\hline
\end{tabular}

Fuente: Elaboración propia

Por otro lado, cabe destacar que, contrariamente a lo esperado, las personas participantes indicaron con claridad que no están dispuestas a sacrificar el compromiso social y los valores a favor del desarrollo profesional y las competencias técnicas y que, más bien, buscan un equilibrio entre unos y otros.

\section{Objetivos profesionales}

Otros datos de contexto que se recogieron para caracterizar la muestra estudiada se refieren a sus objetivos profesionales. Si aproximadamente la quinta parte de los encuestados (21\%) manifestó no tener claros sus objetivos profesionales, la gran mayoría (39\%) indicó los medios de comunicación e internet como entornos en los cuales desearían trabajar. 
Figura 1. Aspiraciones profesionales de la muestra encuestada.

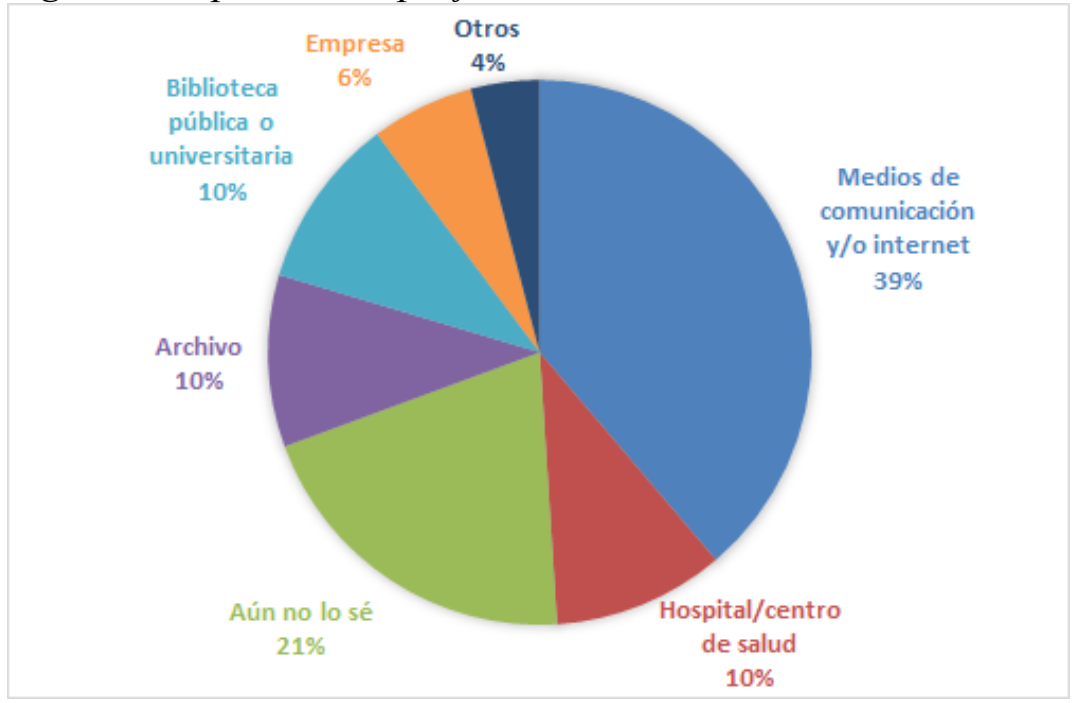

Fuente: Elaboración propia

\section{Actitud ante el proyecto}

Los alumnos describieron su actitud inicial hacia el proyecto pudiendo escoger entre las siguientes opciones: expectación $(16,7 \%)$, rechazo $(8,9 \%)$, aburrimiento $(8,9 \%)$, curiosidad (32,2\%), entusiasmo (10\%), preocupación (12,2\%) e indiferencia $(11,1 \%)$, y mostrando mayoritariamente una actitud de curiosidad. Las respuestas de cada alumno y alumna se codificaron y simplificaron en: actitud negativa (rechazo, aburrimiento, preocupación e indiferencia, 41,1\%), actitud positiva (entusiasmo, 10\%), y actitud neutra (expectación y curiosidad), resultando esta última la más prevalente (48,9\% de las respuestas).

Cuadro 4. Actitud al comienzo del proyecto

\begin{tabular}{|l|c|}
\hline $\begin{array}{l}\text { Actitud al comienzo del } \\
\text { proyecto }\end{array}$ & $\begin{array}{c}\text { \% de } \\
\text { respuestas }\end{array}$ \\
\hline Entusiasmo & 10,0 \\
\hline Expectación & 16,7 \\
\hline Curiosidad & 32,2 \\
\hline Preocupación & 12,2 \\
\hline Indiferencia & 11,1 \\
\hline Aburrimiento & 8,9 \\
\hline Rechazo & 8,9 \\
\hline
\end{tabular}




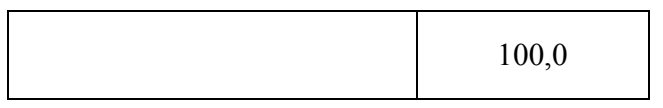

Fuente: Elaboración propia

En una pregunta abierta y complementaria, se destacaron la percepción de falta de organización, la confusión que produjo la necesidad de organizar actividades de forma autónoma, la falta de preparación para ello, así como la incertidumbre que producía tener que relacionarse con personas mayores. Esta sensación de confusión e incertidumbre con respecto a cómo actuar se ha detectado como una característica típica de proyectos de ApS. Riddle (2003) menciona tres etapas en los procesos de aprendizaje y servicio, siendo la primera de "shock emocional y social". Según este autor, los estudiantes, fuera de su entorno académico habitual, experimentarían sensaciones de alienación, incomprensión o asombro ante la magnitud del problema que deben resolver y de incapacidad para afrontarlo. Los resultados de la presente encuesta parecen confirmarlo. Adicionalmente, al comienzo del proyecto los participantes sintieron que la información recibida se contradecía, algo derivado posiblemente de las diferentes partes involucradas en el mismo, y que no estaba clara la relación entre el proyecto y los estudios del Grado en I\&D. Confundir el Aprendizaje y Servicio con "trabajo social fuera de lugar" sería una de las razones de la marginalización de esta estrategia docente en el marco universitario, según Peterson (2009).

\section{Percepción de la experiencia}

Para poder describir la experiencia realizada se definieron una serie de dimensiones, entre las cuales están la existencia de reflexión durante el periodo de actividades, el sentirse a gusto o la percepción de que las actividades formativas realizadas fueran diferentes a las habituales. Los participantes indicaron la frecuencia con la que experimentaron cada una de las situaciones definidas en el curso del proyecto. La respuesta más destacada apunta a que la realización de la actividad se caracterizó por una toma de conciencia de una problemática social como la exclusión informacional de la población mayor. La mayoría de los participantes experimentó siempre o a menudo que las actividades realizadas fueron muy diferentes a las habituales, se sintió a gusto y reflexionó, según puede verse en la Figura 2. Por otro lado, para la mitad o más de los participantes, se 
dieron nunca o solo en ocasiones el poder aclararse sobre la misión de la profesión y el poner en práctica conocimientos de varias asignaturas.

Figura 2. Dimensiones de la experiencia de ApS según la percepción de los participantes

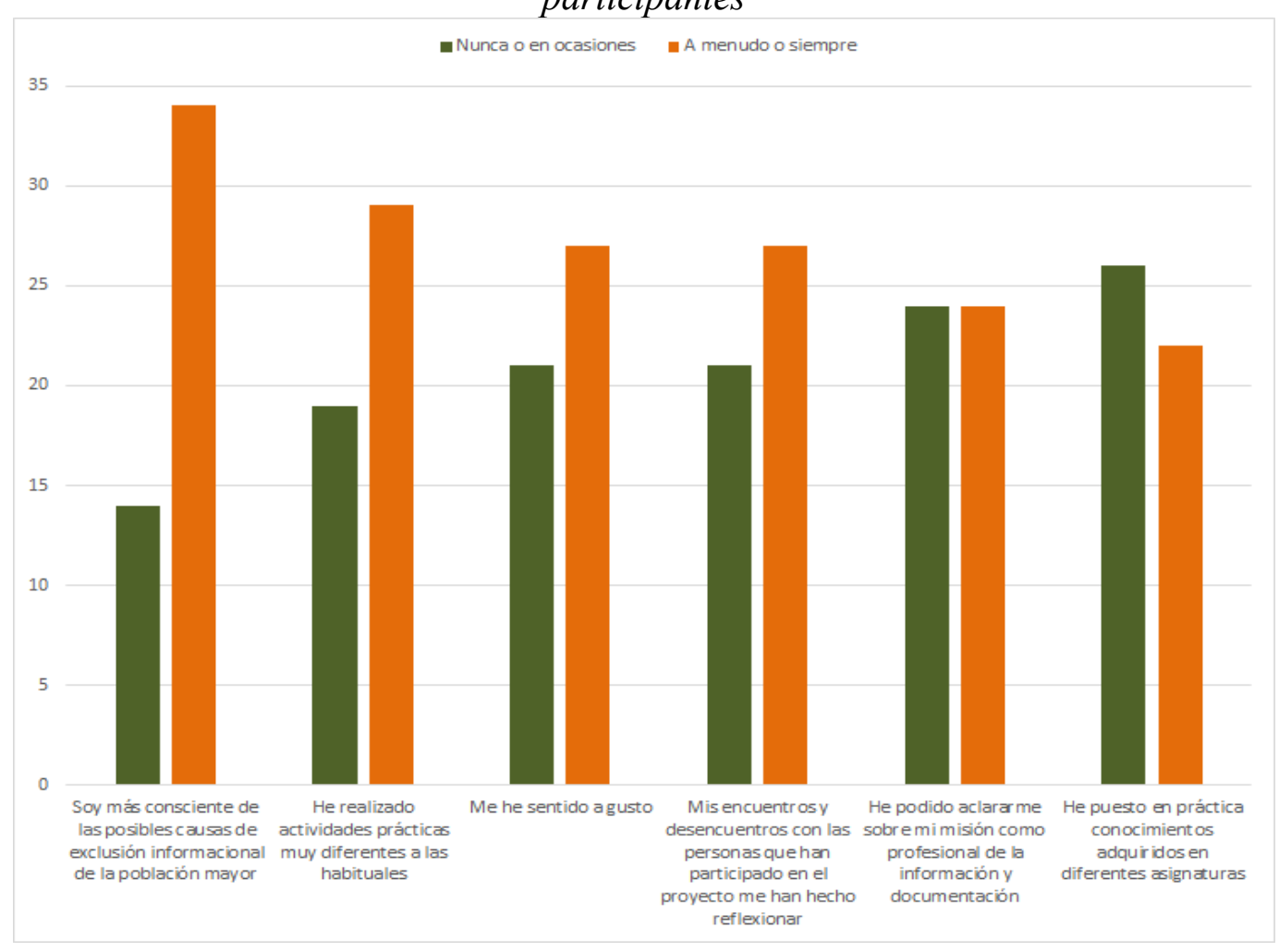

Fuente: Elaboración propia

\section{Competencias desarrolladas}

El avance percibido en nueve competencias, que al principio del proyecto se habían establecido como objetivos para conseguir a través de las actividades, se evaluó en una escala del 1 al 4. En La Figura 3 se ven las evaluaciones del alumnado agregadas en dos bloques ("poco o nada" y "mucho o muchísimo") para mostrar las competencias que, tras la ejecución del proyecto, se perciben como las de mayor avance. El avance más destacado se dio en las competencias necesarias para llevar el trabajo en grupo y en las competencias relacionadas con la comunicación. El avance en la "Prestación de servicios profesionales en base a la empatía" fue positivo para la mayoría de los participantes, aunque no de forma tan destacada como en la primera encuesta realizada en 2018 (Montesi, Portela Filgueira, Ramírez Martín et al., 2019). 
Figura 3. Percepción del alumnado participante acerca del avance en 9 competencias.

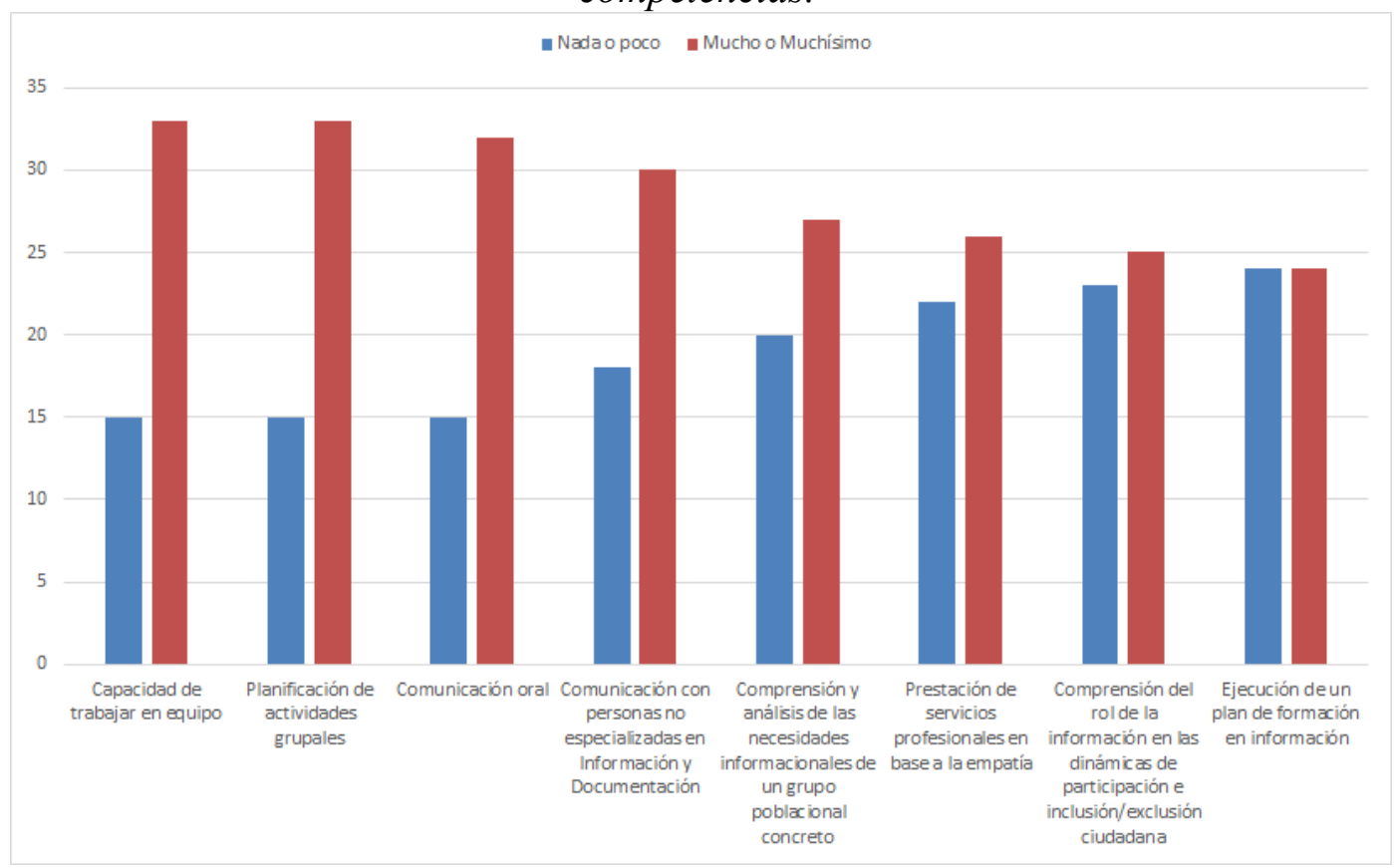

Fuente: Elaboración propia

\section{Pruebas del Chi Cuadrado}

La prueba del Chi Cuadrado se realizó para comprobar si la percepción de la experiencia estaba asociada con un mayor o menor avance en las competencias propuestas en la encuesta. La hipótesis nula pudo rechazarse en varios casos. La dimensión de la experiencia "He podido aclararme sobre mi misión como profesional de la información y documentación" resultó relacionada con todas las competencias de la Figura 3, y la hipótesis nula se rechazó en todos los casos. El valor del Chi-cuadrado de Pearson fue comparativamente más alto para “Comprensión del rol de la información en las dinámicas de participación e inclusión/exclusión ciudadana" $(11,665)$ y para "Prestación de servicios en base a la empatía" (11,733) (Cuadro 5).

\section{Cuadro 5. Valores de significación y fuerza de la correlación entre "He podido} aclararme sobre mi misión... " y las competencias medidas en el proyecto.

\begin{tabular}{|l|c|c|}
\hline Competencias & Valor de $\mathbf{p}$ & Chi-cuadrado de Pearson \\
\hline Capacidad de trabajar en equipo & 0,020 & 5,371 \\
\hline Planificación de actividades grupales & 0,029 & 4,752 \\
\hline Comunicación con personas no especializadas... & 0,001 & 10,101 \\
\hline
\end{tabular}




\begin{tabular}{|l|c|c|}
\hline Comunicación oral & 0,037 & 4,372 \\
\hline Prestación de servicios profesionales en base a la empatía & 0,001 & 11,733 \\
\hline Comprensión y análisis de las necesidades informacionales... & 0,003 & 8,889 \\
\hline Comprensión del rol de la información en las dinámicas... & 0,001 & 11,665 \\
\hline Ejecución de un plan de formación en información & 0,004 & 8,333 \\
\hline
\end{tabular}

Fuente: Elaboración propia

Estos datos y el análisis de las tablas cruzadas que se produjeron como resultados del test ponen de manifiesto que cuando el alumnado, en el transcurso del proyecto, percibe más a menudo que se aclara sobre su misión como profesional, avanza más de lo esperado en todas las competencias, especialmente en aquellas vinculadas a los objetivos cívicos del propio proyecto (empatía y comprensión de la exclusión informacional). Por el contrario, quienes se aclaran menos a menudo sobre su misión profesional, tienden a avanzar en todas las competencias menos de lo esperado. Dicho de otra manera, tener claros los objetivos profesionales permite sacarle mayor provecho a la participación en el proyecto.

La hipótesis nula se rechazó para otras dos dimensiones de la experiencia también. El haber percibido siempre o muy a menudo que las actividades realizadas eran muy diferentes a las habituales resultó en mayores avances, una vez más, en la prestación de servicios en base a la empatía (valor de $\mathrm{p}=0,010$; Chi-cuadrado de Pearson $=6,692$ ) y en la comprensión del rol de la información en las dinámicas... (valor de $\mathrm{p}=0,003$; Chi-cuadrado de Pearson= 8,730). Esto apunta a que, siendo las actividades de ApS muy diferentes a las que normalmente se realizan en ámbitos académicos, devuelven resultados en habilidades como la empatía o la comprensión de fenómenos sociales que no se aprenden fácilmente en las aulas.

Finalmente, el haber puesto en práctica conocimientos adquiridos en diferentes asignaturas muy a menudo o siempre también produjo un mayor avance a varios niveles, concretamente en la comprensión de necesidades informacionales de un grupo poblacional ( $\mathrm{p}$ valor $=0,011$; Chi-cuadrado de Pearson $=6,467$ ); comunicación con personas no especializadas ( $\mathrm{p}$ valor $=0,008$; Chi-cuadrado de Pearson $=6,936$ ); comunicación oral ( $\mathrm{p}$ valor $=0,003$; Chi-cuadrado de Pearson= 
8,759) y capacidad de trabajar en equipo ( $\mathrm{p}$ valor $=0,003$; Chi-cuadrado de Pearson $=8,734)$, es decir, en competencias vinculadas al ámbito de la comunicación y de las interacciones humanas. De alguna manera, el haber aprovechado los conocimientos de diferentes asignaturas mejoró la capacidad de los participantes de interactuar y comunicar tanto con personas mayores como con compañeras y compañeros.

\subsection{Resultados del grupo focal}

\section{Opinión sobre los talleres impartidos}

Las personas mayores que participaron en el grupo focal coincidieron en destacar que los talleres impartidos por alumnos y alumnas de la facultad difieren de los talleres que normalmente se imparten en el centro. En particular, pusieron de relieve el carácter participativo de las clases recibidas por parte del alumnado de la facultad, muy diferente a las clases que se suelen dar en el centro, normalmente impartidas por un solo profesor. En este marco participativo, cada uno de los docentes ha podido atender a las necesidades específicas de cada uno de los alumnos y la docencia en el conjunto ha sido más personalizada. Esto ha permitido observar ciertas cualidades en el alumnado de la UCM, tales como la paciencia, la empatía, el "encanto", las buenas maneras, la accesibilidad, la familiaridad, la cercanía y el entusiasmo. De esta manera, los talleres se han convertido en una especie de reuniones en las que las preguntas y muchas iniciativas salían de los propios asistentes más que de quienes estaban impartiendo el taller. Como consecuencia, el formato del taller no se percibió como estricto y predeterminado sino más flexible y adaptado a las exigencias de los asistentes, repercutiendo en una percepción diferente del tiempo, que ha parecido "volar". Los cursos que impartieron los alumnos UCM se consideraron "vitales" para el día a día, no solo por las temáticas tratadas sino por la forma en la que se imparten y el tipo de interacción humana en la que se basan.

\section{Temáticas de los talleres}

En cuanto a las temáticas de los talleres, en general, se incidió en que enfatizar las tecnologías, sobre todo las tecnologías móviles, es una elección acertada. Las personas mayores participantes, además, insistieron en que les gustaría ver 
muchas más aplicaciones prácticas de las que se vieron, por ejemplo, en el ámbito sanitario y en general todas las de la vida diaria, como comprar billetes de tren o pagar con el móvil. Otra temática importante que se destacó fue la búsqueda de información. Ellos mismos argumentaron que no saber manejar la tecnología de la información y comunicación hoy en día corresponde a cierto grado de analfabetismo.

\section{Experiencia}

Como resultado de los talleres se reiteró que no solo pudieron solucionar dudas, sino que se les plantearon nuevas inquietudes $\mathrm{y}$, al finalizar los talleres, se quedaron con ganas de recibir más clases. El carácter participativo y basado en la atención personalizada de los talleres UCM tuvo repercusiones importantes para los participantes a nivel emocional, pues, por ejemplo, les permitió perder el miedo a los teléfonos y atreverse a experimentar y buscar de forma independiente a partir de los conocimientos de base adquiridos en los cursos.

\section{Aprendizaje de los alumnos}

A las personas mayores asistentes se les preguntó también qué creían que habían aprendido los alumnos y alumnas de la UCM. En su opinión, el alumnado de la UCM había cambiado su imagen de la tercera edad y se tenía que haber llevado una buena sorpresa: "encontrarnos así, con tantas ganas de aprender y capaces de conseguir lo que nos propongamos"... "les hemos sorprendido favorablemente".

En resumen, esta segunda vertiente de la evaluación, desde el punto de vista de las personas mayores, puso de manifiesto el impacto de la iniciativa en la existencia de los asistentes a los talleres. En gran medida los talleres se percibieron como muy efectivos porque se basaban en un modelo participativo e interactivo que permitió al alumnado UCM lucir sus mejores cualidades humanas. Por otro lado, los talleres permitieron a las personas mayores darse a conocer y cambiar la imagen estereotipada de la tercera edad.

\section{Discusión y conclusiones}

En los apartados anteriores, se ha presentado un proyecto de ApS con estudiantes en I\&D de la Universidad Complutense de Madrid y usuarios de Centros de 
Mayores del Ayuntamiento de Madrid, aportando los resultados de una encuesta con estudiantes y de un grupo focal con las personas mayores participantes. Desde el punto de vista docente, el ApS como metodología docente en I\&D ha permitido diversificar las estrategias formativas respondiendo, en este sentido, a la demanda de una formación variada manifestada en la encuesta por parte del alumnado participante (Cuadro 3). Para el alumnado encuestado, la necesidad de desarrollo profesional y de adquisición de competencias técnicas se percibieron como igualmente importantes que el compromiso social y los valores, muy propios de la filosofía del ApS, poniendo de manifiesto ciertas carencias en el planteamiento de los estudios de I\&D que mencionábamos en la introducción, muy centrados en la adquisición de técnicas, y la capacidad de aporte en este sentido de la metodología de ApS.

Desde el punto de vista formativo, el alumnado valoró la experiencia destacando ciertas características de la misma y el avance conseguido en determinadas competencias. En cuanto a las características que se percibieron como destacadas en la experiencia de ApS, el alumnado del grado en I\&D señaló en particular dos: (a) haber tomado conciencia de una problemática social como la exclusión informacional de la población mayor, y (b) haber realizado actividades diferentes respecto a las más habituales en su formación. El hecho de haber percibido las actividades realizadas como diferentes a las habituales resultó estar relacionado, además, con el avance en dos competencias: la empatía y la comprensión del rol de la información en las dinámicas de inclusión/exclusión ciudadana. En este sentido, la participación en el proyecto ha permitido comprender una realidad que en las aulas solo puede abordarse desde una perspectiva teórica, desempeñando un rol complementario en la formación de los estudiantes participantes y corroborando el argumento de la complementariedad entre formación tradicional y metodología ApS ya propuesto por Bloomquist (2015).

Según los resultados de la encuesta, las competencias que más se desarrollaron fueron transversales, destacando las relacionadas con la comunicación y la interacción en contextos grupales que, en el caso del ApS, incluyen también los actores comunitarios (Blodgett, 2017). Aunque menos de la mitad de los estudiantes pudieron aclararse sobre su misión como profesionales en el 
transcurso del proyecto (Figura 2), y todavía la quinta parte de los participantes no tiene claros sus objetivos profesionales (Figura 1), desarrollando competencias comunicativas, la experiencia apoyó a un sector importante de los mismos en sus ambiciones profesionales, pues el 39\% de estos mencionó el sector de la comunicación (medios de comunicación e internet) como objetivo profesional. Asimismo, la comprensión de necesidades informacionales de un grupo poblacional, la comunicación con personas no especializadas, la comunicación oral y la capacidad de trabajar en equipo, todas competencias vinculadas al ámbito de la comunicación y de las interacciones humanas, se vieron impulsadas cuando se percibió que se ponían en práctica conocimientos adquiridos en diferentes asignaturas, indicando las implicaciones a nivel comunicativo de una formación interdisciplinaria y/o la ventaja de participar en proyectos de este tipo una vez cursadas ya varias asignaturas del plan de estudios.

Solo la mitad aproximadamente del alumnado participante sintió haberse aclarado sobre su misión profesional siempre o muy a menudo en el transcurso del proyecto, algo que pone de manifiesto cierta confusión con respecto a las titulaciones en I\&D, incluso por parte del alumnado matriculado en las mismas. Este resultado es particularmente importante si tenemos en cuenta que el aclararse o no sobre la misión profesional puede repercutir positiva o negativamente en el avance en todas las competencias trabajadas (Cuadro 5). Es difícil determinar las causas de esta incertidumbre o confusión que puede achacarse a la percepción general de la disciplina, a la planificación del proyecto o a características individuales de los alumnos participantes. Las propias características del proyecto y de las interacciones universidad-comunidad pueden producir esta confusión, exigiendo asumir roles múltiples, fluidos y cambiantes (Sieh y Frank, 2018), y una buena preparación del alumnado con respecto a los diferentes roles que debe asumir en el transcurso de un proyecto como el presente puede fomentar la consecución de los objetivos planteados. Cabe añadir aquí una referencia a las ediciones posteriores del proyecto, la del curso 2019-20, que se interrumpió por la pandemia de Covid-19, y la del curso 2020-21, en la que las modalidades de interacción con la población mayor se han tenido que reajustar a las medidas de distanciamiento social, demostrando la necesidad de adaptar roles e interacciones 
a una realidad cambiante. Significativamente, las encuestas de seguimiento del alumnado que se realizaron en abril de 2020, en plena pandemia, pusieron de manifiesto, frente a los datos que presentamos aquí, una intensificación de la experiencia en términos de reflexión y toma de consciencia de las problemáticas sociales trabajadas, y un avance mayor en competencias como el trabajo en equipo y la empatía, a pesar de la imposibilidad de llevar a cabo los talleres planificados (Cristóbal Querol, Ganduz Ávila, García Moreno et al., 2020a). La situación de pandemia ha hecho que el alumnado se aclare sobre su misión profesional y lo que puede aportar a la comunidad de manera más contundente que en las ediciones anteriores, subrayando la relevancia para la formación profesional del contacto con la realidad social.

Desde el punto de vista de las personas mayores, el impacto del proyecto se midió de forma cualitativa con un colectivo reducido del que formaba parte también una representante de la dirección del centro. Si nos centramos en el significado de esta experiencia por encima de los resultados, según sugieren Sieh y Frank (2018), las ideas que se destacaron incluyen la participación, la calidad humana y la vitalidad de los contenidos impartidos en los talleres. En cuanto a la participación, cabe reiterar que la mutualidad de las interacciones alumnado-comunidad es un elemento básico de la metodología del ApS, según indicábamos en la introducción. Los talleres de alguna manera se co-impartieron, porque las personas mayores recibieron atención personalizada y adaptada a las necesidades que iban expresando. Con respecto a la calidad humana del alumnado participante, Cronley et al. (2015) argumentan que la dedicación, la profesionalidad y el trabajo del alumnado son elementos fundamentales para las organizaciones participantes a la hora de decidir si participar o no en un proyecto de ApS. Por lo que concierne a la vitalidad de los contenidos, este concepto se enfatizó en el marco de la vida diaria, corroborando la idea de que, posiblemente, la disciplina debería ampliar sus horizontes más allá de las bibliotecas, los centros de documentación y los archivos, para dar cabida a problemas informacionales de mucho más amplio espectro. Finalmente, el impacto de mayor trascendencia social quizás haya sido dar a las personas mayores una oportunidad de darse a conocer y de desmitificar la imagen estereotipada de la tercera edad. 
Para concluir, aunque los datos recopilados hasta el momento acerca de la institución y del profesorado no están suficientemente sistematizados (Cristóbal Querol, García Moreno, Montesi et al., 2020), la experiencia etnográfica que mencionábamos en la metodología nos permite añadir que un proyecto como este tiene implicaciones y conexiones naturales con la investigación. Desafortunadamente, es aún poco reconocida la acción transformadora que se lleva a cabo a través de estos proyectos, quizás por la ausencia de un marco evaluador claro. Según Arocena et al. (2018), los desafíos de sostenibilidad de la sociedad actual exigen no solo que la investigación aborde nuevos problemas, sino que incluya en su agenda problemas normalmente no tratados, especialmente aquellos cargados de connotaciones de valor como las disparidades existentes entre países ricos y países en vías de desarrollo. Queda de manifiesto la necesidad de documentar el impacto de este tipo de iniciativas y sobre todo su vinculación a dimensiones más reconocidas de la actividad académica, especialmente la investigación.

\section{Referencias bibliográficas}

Abad Alcalá, L., et al. (2017) Administración electrónica y trámites online: hacia la autonomía y empoderamiento de las personas mayores. El profesional de la información, 26(1), 34-42.

Abellán García, A., et al (2019). Un perfil de las personas mayores en España, 2019. Indicadores estadísticos básicos. Informes Envejecimiento en red $n^{\circ}$ 22. Madrid. Recuperado de http://envejecimiento.csic.es/documentos/documentos/enredindicadoresbasicos2019.pdf

Agencia Nacional de Evaluación de la Calidad y Acreditación (ANECA). (2004). Libro Blanco. Título de Grado en Información y Documentación. Recuperado de http://www.aneca.es/var/media/150424/libroblanco_jun05_documentacion .pdf

Amat, A. F. (2011) Usando la web 2.0 para informarse e informar. Una experiencia en educación superior. Teoría de la Educación. Educación y Cultura en la Sociedad de la Información (TESI), 12(1), 145-166. 
American Library Association (2009). Final Core Competences Statement. Recuperado de http://www.ala.org/educationcareers/sites/ala.org.educationcareers/files/co ntent/careers/corecomp/corecompetences/finalcorecompstat09.pdf

Angel, C. M. (2016). Collaboration among Faculty Members and Community Partners: Increasing the Quality of Online Library and Information Science Graduate Programs through Academic Service-Learning. Journal of Library \& Information Services in Distance Learning, 10(1-2), 4-14.

Aramburuzabala, P. (2013). Aprendizaje-Servicio: Una herramienta para educar desde y para la justicia social. RIEJS Revista Internacional de Educación para la Justicia Social, 2(22), 5-11.

Arocena, R., Göransson, B., Sutz, J. (2018). Towards making research evaluation more compatible with developmental goals. Science and Public Policy, 46(2), 210-218.

Ayuntamiento de Madrid. Dirección General De Personas Mayores Y Servicios Sociales (2019). Fomento de la imagen adecuada del envejecimiento. Recuperado de https:/www.madrid.es/UnidadesDescentralizadas/Mayores/Especial\%20in formativo/Madrid, $\% 20$ ciudad $\% 20$ amigable/Fomento $\% 20 \mathrm{de} \% 201 \mathrm{a} \% 20 \mathrm{ima}$ gen $\% 20$ adecuada $\% 20$ del $\% 20$ envejecimiento $2 . p d f$

Batlle, R. (s. f.). Aprendizaje Servicio. Definición. Recuperado de https://roserbatlle.net/aprendizaje-servicio/

Batlle, R., Escoda, E. (Coord). (2019). 100 buenas prácticas de aprendizajeservicio. Inventario de experiencias educativas con finalidad social. Salamanca, Valencia, Madrid: Santillana, Red española Aprendizaje Servicio, Escuela Técnica Superior de Ingenieros de Minas y Energía

Blodgett, J. (2017). Taking the class out of the classroom: Libraries, literacy, and service learning. En: Macdonell, P. (Ed.). The Experiential Library: Transforming Academic and Research Libraries through the Power of Experiential Learning. Cambridge, MA, Chandos, p. 43-52.

Bloomquist, C. (2015). Reflecting on reflection as a critical component in service learning. Journal of Education for Library and Information Science, 56(2), 169-172.

Chupp, M. G., Josephj, M. L. (2010). Getting the most out of service learning: Maximizing student, university and community impact. Journal of Community Practice. 18(2-3), 190-212. 
Colón-Aguirre, M. (2017) Service learning for improvement of customer service education in LIS. Education for Information, 33 (3), 171-185.

Cristóbal Querol, G. D., García Moreno, M., Montesi, M., Ovalle Perandones, M., Portela Filgueiras, I., Ramírez Martín, S. M., ... \& Villaseñor Rodríguez, I. (2020). Experiencia Aprendizaje y Servicio en el Grado en Información y Documentación. Recuperado de https://eprints.ucm.es/id/eprint/62031/

Cristóbal Querol, G.D., Ganduz Ávila, A.M., García Moreno, M.A., Montesi, M., Ovalle Perandones, M.A., Portela Filgueira, I., Ramírez Martín, S., Ramos Simón, F., Razquin Zazpe, P., Sacristán Sánchez, M., Villaseñor Rodríguez, I. (2020a). Actividades de formación en competencias informacionales en Centros de Mayores del Ayuntamiento de Madrid (Memoria proyecto ApS UCM). Universidad Complutense de Madrid. (No publicado). Recuperado de https://eprints.ucm.es/id/eprint/61591/

Cronley, C., Madde, E., Davis, J. B. (2015). Making service-learning partnerships work: Listening and responding to community partners. Journal of Community Practice, 23(2), 274-289.

Espelt, C., Mañá, T. (2011). El valor añadido de las prácticas externas para los estudiantes y las instituciones: La experiencia de la Facultad de Biblioteconomía y Documentación de Barcelona. En: World Library and Information Congress: 77th IFLA General Conference and Assembly, 1318 Agosto, 2011, San Juan, Puerto Rico, 120.

Folgueiras Bertomeu, P., Luna González, E., \& Puig Latorr, G. (2013). Aprendizaje y servicio: estudio del grado de satisfacción de estudiantes universitarios. Revista de Educación, 362, (septiembre-diciembre), 159185.

Frank, A. I., Sieh, L. (2016). Multiversity of the twenty-first century-examining opportunities for integrating community engagement in planning curricula. Planning Practice \& Research. 31(5), 513-532.

Fundación Telefónica. (2018). Sociedad Digital en España 2017. Fundación Telefónica. Recuperado de https://www.fundaciontelefonica.com/culturadigital/publicaciones/sociedad-digital-en-espana-2017/625/

Fundación Telefónica. (2019). Sociedad Digital en España 2018. Fundación Telefónica. Recuperado de https://www.fundaciontelefonica.com/culturadigital/publicaciones/sociedad-digital-en-espana-2018/655/ 
González Oñate, C., Fanjul Peyró, C. (2018) Aplicaciones móviles para personas mayores: un estudio sobre su estrategia actual. Aula Abierta, 47(1), 107112. DOI: https://doi.org/10.17811/rifie.47.1.2018.107-112

González -Teruel, A., Andreu-Ramos, C. (2013). Investigación del comportamiento informacional a través del análisis de redes sociales. $E l$ profesional de la información, 22(6), 522-528.

Grup de Recerca en Educació Moral, Centre Promotor de 1'Aprenentatge Servei (2019). Blog de l'Aprenentatge Servei. Recuperado de https://apsvalors.wordpress.com/

James, J. H., Logan, K. (2016). Documenting the community impact of servicelearning coursework: Theoretical and practical considerations. Partnerships: A Journal of Service-Learning and Civic Engagement, 7(2), 17-36.

Jardine, F. (2016). The role of students in diversity and inclusion in library and information science. En: Perspectives on libraries as institutions of human rights and social justice. Emerald Group Publishing Limited. 394-416.

Juarros, V. M., Moreno, J., Bennasar, F. N. (2012). Modelos educativos para la gestión de la información en educación superior: una experiencia de curación de contenidos como estrategia metodológica en el aula universitaria. Edutec. Revista Electrónica de Tecnología Educativa, 42, (diciembre), a218.

Kott, K. (2016). Service Learning in Academic Libraries in the United States. En: Nutefal, J. E. (ed.). Service learning, information literacy, and libraries. Santa Barbara, California: ABC-CLIO, p. 120.

Lim, S., Bloomquist, C. (2015). Distinguishing service learning from other types of experiential learning. Education for Information, 31(4), 195-207.

Llorente Barroso, C., Viñarás Abad, M., Sánchez Valle, M. (2015). Mayores e internet: la Red como fuente de oportunidades para un envejecimiento activo. Comunicar, 45(23), 29-36.

Marullo, S., Edwards, B. (2000). From charity to justice: The potential of university-community collaboration for social change. American Behavioral Scientist, 43(5), 895-912.

Miettinen, R., Tuunainen, J., Esko, T. (2015). Epistemological, artefactual and interactional-institutional foundations of social impact of academic research. Minerva, 53(3), 257-277. 
Miguel, I., Da Luz, H. A. (2017). Internet use for active aging: A systematic literature review. En: 12th Iberian Conference on Information Systems and Technologies (CISTI), 21-24 June 2017, Lisboa, 2444-2449. DOI: 10.23919/CISTI.2017.7975697.

Mizzy, D., Hayslett, M. (2016). Data librarianship: A day in the Life-Science Edition. En: L.M. Kellam, K. Thompson (Ed.), Datalibrarianship: The academic data librarian in theory and practice. Chicago: Association of College and Research Libraries, 335-351.

Montague, R. A., Wolske, M., Larkaee, B. (2009). Service learning from three perspectives. En: Roy, L., Jensen, K., Meyers, A. H. (Ed.). Service learning: Linking library education and practice. Chicago: American Library Association, 32-46.

Montesi, M., Villaseñor Rodríguez, I. (2018). El impacto social de las instituciones de educación superior Un estudio de caso con la Universidad Complutense de Madrid. Información, cultura y sociedad, 39, (diciembre), p. 37-60.

Montesi, M., Portela Filgueira, I., Ramírez Martín, S., Villaseñor Rodríguez, I. (2019). "Aprendizaje y Servicio (ApS) en los estudios de Información y Documentación: resultados preliminares de un proyecto UCM. En: Simeão, E., Cuevas Cerveró, A., Botelho, R., \& Gómez-Hernández, J. A. (eds.). Competência em Informação e Políticas para a Educação Superior. Estudos Hispano-Brasileiros, pp. 162-175. Recuperado de https://eprints.ucm.es/id/eprint/59952/25/volumen1.pdf

Montiel Overall, P. (2010). The effect of service learning on LIS students' understanding of diversity issues related to equity of access. Journal of Education for Library and Information Science, 51(4), 251-266.

Most, L. R. (2011). Hands on from a distance: The community-embedded learning model contextualizes online student coursework. Journal of Education for Library and Information Science, 52(4), 295-304.

Naciones Unidas (2020). Policy Brief: The Impact of COVID-19 on older persons. Recuperado de https://unsdg.un.org/sites/default/files/2020-05/PolicyBrief-The-Impact-of-COVID-19-on-Older-Persons.pdf

Nutefall, J. E. (Ed.). (2016). Service learning, information literacy, and libraries. Santa Barbara, California: ABC-CLIO.

Ochoa, E. M. (Ed). (2014). Universidades solidarias Programa de apoyo y fortalecimiento de proyectos de aprendizaje-servicio solidario para 
universidades de América Latina. Buenos Aires: CLAYSS. Recuperado de https://www.clayss.org.ar/04 publicaciones/UniversidadesSolidarias.pdf

Ontsi (2018). Perfil sociodemográfico de los internautas. Análisis de datos INE 2018. Observatorio Nacional de las Telecomunicaciones y la Sociedad de la Información. Recuperado de https://www.ontsi.red.es/es/estudios-einformes/perfil-sociodemografico-de-los-internautas-datos-ine-2018

Pérez-Lorenzo, B., Vianello Osti, M., Pacios, A. R. (2013). El Practicum del Grado en Información y Documentación: La experiencia en la UC3M. Anales de Documentación, 16 (2). DOI:

https://doi.org/10.6018/analesdoc.16.2.180381.

Peterson, L. (2009). A brief history of Service Learning in LIS. En: Roy, L., Jensen, K., Meyers, A. H. (Ed.). Service learning: Linking library education and practice. Chicago: American Library Association, 1-4.

Pino Juste, M. R., Soto Carballo, J. G,. Rodríguez López, B. (2015). Las personas mayores y las TIC. Un compromiso para reducir la brecha digital. Pedagogía Social. Revista Interuniversitaria, 26, 337-359.

Riddle, J. S. (2003). Where's the library in service learning?: Models for engaged library instruction. The Journal of Academic Librarianship, 29(2), 71-81.

Rodríguez-Gallego, M. R. (2014). El Aprendizaje-servicio como estrategia metodológica en la Universidad. Revista Complutense de Educación, 25(1), 95-113.

Rodríguez-Gallego, M. R., Ordóñez-sierra, R. (2015). Una experiencia de aprendizaje servicio en comunidades de aprendizaje. Profesorado. Revista de curriculum y formación del profesorado, 19(1), 314- 333.

Roy, L., Jensen, K., Meyers, A. H. (Ed.). (2009). Service learning: Linking library education and practice. Chicago: American Library Association.

Rubio I Serrano, L., Prats Gil, E., Gómez, L. (2013). Universidad y sociedad. Experiencias de aprendizaje servicio en la universidad. Colección Educación y Comunidad, 8. Barcelona: Universitat de Barcelona.

Sánchez-Esparza, M. (2018). Alfabetización Digital con smartphones, un medio para reducir la soledad en los mayores. En: Casas, P., Paramio, G., Castro, A. (Ed.). Educación y Comunicación Mediada por las Tecnologías: tendencias y retos de investigación. Sevilla: Egregius Ediciones, 29-40. 
Sieh, L., Frank, A. I. (2018). Designing impact evaluation for students' engagement with communities in planning education. Journal of Planning Education and Research, (October), DOI: 10.1177/0739456X18807287.

Tapia, M. N. (2008). Aprendizaje y servicio solidario. Buenos Aires: Ciudad Nueva.

Tapia, M. N. Y Montes, R. (eds.) (2016). Herramientas prácticas para desarrollar un proyecto de aprendizaje-servicio solidario. Ciudad Autónoma de Buenos Aires: CLAYSS.

Tejada-Artigas, C. M., Chacón Jarén, S. \& Moreiro-González, J. A. (2014). Mercado de trabajo en información y documentación y crisis económica en España: una aproximación a partir de las ofertas publicadas en IweTel entre 2008 y 2013. BiD, 32, (junio). DOI: $10.1344 /$ BiD2014.32.15

Thompson, V. L. S., Hood, S. M. (2016). Academic and community partnerships and social change. En: The crisis of race in higher education: A day of discovery and dialogue. Bingley, UK: Emerald Group Publishing Limited, 127-149.

Trencher, G., Tereda, T., Yarime, M. (2015). Student participation in the cocreation of knowledge and social experiments for advancing sustainability: experiences from the University of Tokyo. Current Opinion in Environmental Sustainability, 16, 56-63.

Trencher, G., et al. (2013). Beyond the third mission: Exploring the emerging university function of co-creation for sustainability. Science and Public Policy. 41(2), 151-179.

Villaseñor-Rodríguez, I. (2017) Estudios de usuarios de información. Diseño metodológico e informe final. Barcelona: Editorial UOC.

Yu, T. K., \& Chao, C. M. (2014). Assessing older adults' information technology ability: The development of a multiple item scale. International Journal of Human-Computer Interaction, 30(6), 435-445.

\section{Agradecimientos}

El proyecto se ha financiado en el marco de los Proyectos de Innovación y Mejora de la Calidad Docente 2018, Vicerrectorado de Calidad, Universidad Complutense de Madrid, y de los Proyectos de Aprendizaje y Servicio, 
convocatoria 2018-19, de la Delegación del Rectorado para la Diversidad y el Medioambiente, Universidad Complutense de Madrid.

\section{Nota contribución autoral}

Las tres autoras participaron en la ejecución del proyecto del que trata el artículo y realizaron conjuntamente la parte de introducción y conclusiones del trabajo. El diseño y análisis de la encuesta corresponden a M. Montesi.

\section{Nota del editor}

El editor responsable para la publicación de este artículo fue Mario Barité. 